\title{
Fragmentation Function Measurements at Belle
}

\author{
Akio Ogawa ${ }^{1}$, Matthias Grosse Perdekamp ${ }^{2}$ and Ralf Seidl ${ }^{1,2}$ \\ 1- RIKEN Brookhave Research Center \\ Upton, NY 11973-5000, USA \\ 2- University of Illinois at Urbana-Champaign \\ 1110 W Green Street, Urbana, IL 61801, USA
}

\begin{abstract}
In order to measure the quark transverse spin distribution of the nucleon one has to combine it with another chiral-odd object. This can for example be the Collins fragmentation function or the Interference fragmentation function. At the KEKB $e^{+} e^{-}$ collider the Belle experiment has published the first measurement of the Collins fragmentation function with a data sample of $29 \mathrm{fb}^{-1}$. In an updated analysis a data sample of $547 \mathrm{fb}^{-1}$ has been analyzed reducing the statistical errors significantly.
\end{abstract}

\section{Introduction}

At leading twist 3 quark distribution functions (DF) in the nucleon are present; the experimentally well known unpolarized quark DF, the experimentally less known quark helicity DF and the so far undetermined transversity DF. The latter cannot be measured in inclusive DIS due to its chiral-odd nature, since all possible interactions are chiral-even for nearly massless quarks. Therefore one needs an additional chiral-odd function in the cross section to access transversity. This can be achieved either by an anti quark transversity DF in double transversely polarized Drell-Yan processes or, alternatively, one can have a chiral-odd fragmentation function in semi-inclusive deep inelastic scattering (SIDIS) or hadroproduction. The most prominent chiral-odd fragmentation function is the Collinsfunction [2], which measures the azimuthal distribution of an unpolarized hadron around the axis of the transversely polarized quark's momentum. A second chiral-odd fragmentation function is the Interference fragmentation function [3], where one meeasures the azimuthal distribution of a hadron pair around the quark axis. In SIDIS nonzero Collins asymmetries have been measured [4], but in order to extract transversity one has to obtain the fragmentation function separately. This can be acchieved in a $e^{+} e^{-}$collider.

\section{The Belle experiment}

The Belle [5] experiment at the asymmetric $e^{+} e^{-}$collider KEKB at Tsukuba, Japan, is mainly dedicated to the study of $\mathrm{CP}$ violation in $\mathrm{B}$ meson decays. Its center of mass energy is tuned to the $\Upsilon(4 S)$ resonance at $\sqrt{s}=10.58 \mathrm{GeV}$. Part of the data was also recorded 60 $\mathrm{MeV}$ below the resonance. Originally only these off-resonance events were studied in order to measure spin dependent fragmentation functions (FF). Since one selects only two-jet-like events requiring a thrust value larger than 0.8 only less than $2 \%$ of the B events remain in the on-resonance data sample. Therefore, it is possible to include also that data sample in the analsys. In total an integrated luminosity of $547 \mathrm{fb}^{-1}$ has been analyzed. The aerogel Čerenkov counter, time-of-flight detector and the central drift chamber enable a good particle identification and tracking, which is crucial for these measurements. Using the information from the silicon vertex detector, one selects tracks originating from the interaction region and 

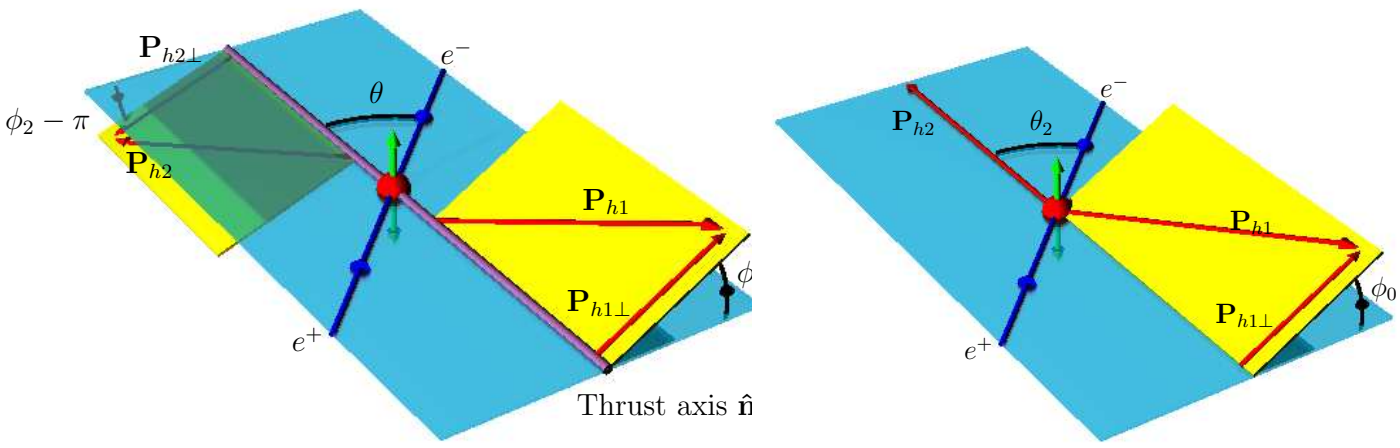

Figure 1: Description of the azimuthal angles $\phi_{0}, \phi_{1}$ and $\phi_{2}$ relative to the scattering plane defined by the lepton axis and either the thrust axis $\hat{n}$ or the momentum of the $2^{\text {nd }}$ hadron $P_{h 2}$.

thus reducing the contribution of hadrons from heavy meson decays. To reduce the amount of hard gluon radiative events, a thrust value larger of 0.8 and a virtual photon momentum in the two-hadron center-of-mass system below $3.5 \mathrm{GeV}$ is required. In such a 2-jet topology the thrust axis can be used as an approximation of the original quark direction. We also require that the fractional energy $z \stackrel{C M S}{=} 2 E_{h} / Q>0.2$ in order to significantly reduce the contribution of pions arising from the vector meson decays or of those assigned to a wrong hemisphere.

\section{Collins FF}

The Collins effect occurs in the fragmentation of a transversely polarized quark with polarization $S_{q}$ and 3-momentum k into an unpolarized hadron of transverse momentum $P_{h \perp}$ with respect to the original quark direction. According to the Trento convention [6] the number density for finding an unpolarized hadron $\mathrm{h}$ produced from a transversely polarized quark $\mathrm{q}$ is defined as:

$$
D_{h q^{\uparrow}}\left(z, P_{h \perp}\right)=D_{1}^{q}\left(z, P_{h \perp}^{2}\right)+H_{1}^{\perp q}\left(z, P_{h \perp}^{2}\right) \frac{\left(\hat{\mathbf{k}} \times \mathbf{P}_{h \perp}\right) \cdot \mathbf{S}_{q}}{z M_{h}},
$$

where the first term describes the unpolarized FF $D_{1}^{q}\left(z, P_{h \perp}\right)$, with $z \stackrel{C M S}{=} \frac{2 E_{h}}{Q}$ being the fractional energy the hadron carries relative to half of the center of mass system (CMS) energy Q. The second term, containing the Collins function $H_{1}^{q \perp}\left(z, P_{h \perp}^{2}\right)$, depends on the spin of the quark and thus leads to an asymmetry as it changes sign under flipping the quark spin. The vector product can accordingly be described by a $\sin (\phi)$ modulation, where $\phi$ is the azimuthal angle spanned by the transverse momentum and the plane defined by the quark spin and its momentum. In $e^{+} e^{-}$hadron production the Collins effect can be observed by a combined measurement of a quark and an anti quark fragmentation. Combining two hadrons from different hemispheres in jetlike events, with azimuthal angles $\phi_{1}$ and $\phi_{2}$ as defined in Fig. 1, would result in a $\cos \left(\phi_{1}+\phi_{2}\right)$ modulation. 
In the CMS these azimuthal angles are defined between the transverse component of the hadron momenta with regard to the thrust axis $\hat{n}$ and the plane spanned by the lepton momenta and $\hat{n}$. The comparison of the thrust axis calculations using reconstructed and generated tracks in the MC sample shows an average angular separation between the two of $75 \mathrm{mrad}$ with a a root mean square of $74 \mathrm{mrad}$. Due to that small biases in one of the reconstruction methods used could arise and were studied as discussed later. Following reference [7] one either computes the azimuthal angles of each pion relative to the thrust axis which results in a $\cos \left(\phi_{1}+\phi_{2}\right)$ modulation or one calculates the azimuthal angle relative to the axis defined by the $2^{\text {nd }}$ pion which results in a $\cos \left(2 \phi_{0}\right)$ modulation. While the first method directly accesses moments of the Collins functions, the second method contains a convolution integral of the Collins FF over possible transverse momenta of the hadrons.

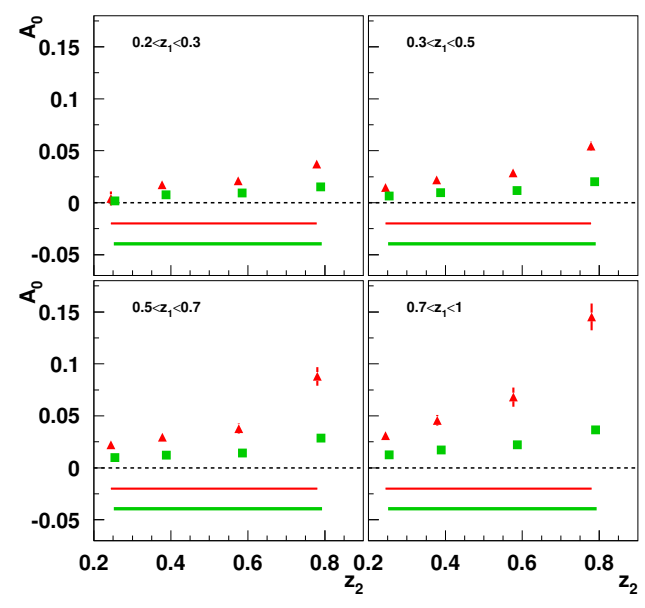

Figure 2: Light quark (uds) $A_{0}$ asymmetries as a function of $z_{2}$ for the $4 z_{1}$ bins. The UL data is described by the triangles, its systematic error being the top error band while the UC data is described by the squares and its systematics by the lower error band.

\section{$1.1 \quad$ Measured asymmetries}

We measure the azimuthal asymmetries $N(2 \phi) /\left\langle N_{0}\right\rangle$, where $N(2 \phi)$ denotes the number of hadron pairs in bins of either $2 \phi_{0}$ or $\phi_{1}+\phi_{2}$ and $\left\langle N_{0}\right\rangle$ is the average number of hadron pairs in that particular bin. The main backgrounds, producing similar azimuthal asymmetries as the Collins effect, are the radiation of soft gluons and acceptance effects. The gluonic contribution is proportional to the unpolarized FF and is independent of the charge of the hadrons. Consequently taking the ratio of the normalized distributions for unlike-sign over like-sign pairs the gluonic distributions cancel in the leading order:

$$
\begin{aligned}
\frac{R_{\alpha}^{U}}{R_{\alpha}^{L}} & :=\frac{\left.\frac{N(2 \phi)}{\left\langle N_{0}\right\rangle}\right|_{\text {Unlikesign }}}{\left.\frac{N(2 \phi)}{\left\langle N_{0}\right\rangle}\right|_{\text {Likesign }}} \\
& \approx 1+\frac{\sin ^{2} \theta}{1+\cos ^{2} \theta}\left(F\left(\frac{H_{1}^{\perp, f a v}}{D_{1}^{\text {fav }}}, \frac{H_{1}^{\perp, \text { dis }}}{D_{1}^{\text {dis }}}\right)+\mathcal{O} f\left(Q_{T}, \alpha_{S}\right)^{2}\right) \cos (2 \phi)
\end{aligned}
$$

where $\theta$ is either the angle between the colliding leptons and the produced hadron or the thrust axis for methods $\alpha=0,12$, respectively. Favored and disfavored FF (fav,dis) describe the fragmentation of a light quark into a pion of same or opposite charge sign. Obviously also acceptance effects cancel in the double ratios. The latter are fit by the sum of a constant term and a cosine modulation, $B_{\alpha}+A_{\alpha} \cos (2 \phi)$. The double ratios of unlike sign over like sign pairs showed the existence of the Collins effect and gave a hint about the overall 
magnitude [8]. As suggested in [9], measuring also double ratios containing any combination of charged pion pairs reveals additional information on the ratio of the favored and disfavored Collins functions. The $A_{0}$ results with the updated statistics are shown as function of the fractional energies $z_{1}$ and $z_{2}$ in Fig.2. The $A_{12}$ results are similar and all are consistent with the previously published data. The data has been corrected for the contribution of charmed hadron decays. A nonzero asymmetry is visible for both double ratios, and the $\mathrm{U} / \mathrm{C}$ are about $40 \%$ of the $\mathrm{U} / \mathrm{L}$ results. The data shows a rising behavior with rising fractional energy z for both results. Several systematic cross-checks of the analysis method were performed and the differences in the results are quoted as systematic uncertainties: Instead of double ratios we used the subtraction method for the unlike from the like sign or charged pion asymmetries; the constant fit to the double ratios obtained in MC (without a Collins contribution) together with its statistical error and a similar fit to double ratios of positively charged over negatively charged pion pair data were assigned as systematic error. The differences to the results when fitting the double ratios also with higher order azimuthal modulations were added to the systematic errors. All contributions to the systematic errors were added in quadrature. The presented measurement represents a substantial update of the data sample used to obtain the first evidence of the Collins effect and will help to disentangle the favored to disfavored Collins function ratio.

\section{References}

[1] Slides:

http: //indico. cern. ch/contributionDisplay . py? contribId=149\&sessionId=4\&conf Id $=9499$

[2] J. C. Collins: Nucl. Phys B396(1993):161.

[3] R. L. Jaffe, X. m. Jin and J. a. Tang: Phys. Rev. D57(1998):5920

[4] A. Airapetian et al.(Hermes) Phys. Rev. Lett.94(2005)012002.

[5] A. Abashian et al.(Belle): Nucl. Instrum. Meth.A479(2002)117.

[6] A. Bacchetta, U. D'Alesio, M. Diehl, A. Miller: Phys. Rev. D70(2004):117504.

[7] D. Boer, R. Jakob, P. J. Mulders: Phys. Let. B 424(1998):143.

[8] R. Seidl, et al.(Belle): Phys. Rev. Lett. 96(2006):232002.

[9] A. Efremov, K. Goeke and P. Schweitzer: [arXiv:hep-ph/0603054]. 